\title{
DYNAMICS OF THE ASYMETRIES
}

\section{AT GALACTIC CENTERS}

\author{
F. MASSET AND M. TAGGER
}

Service d'Astrophysique (CNRS URA 2052), CEA-Saclay, 91191

Gif/Yvette Cedex, France

\section{Introduction}

Bars in galaxies are frequently found to be off-centered, so that the center of the bar does not coincide with the center of rotation, with a difference which typically amounts to several tens of parsecs. This is clearly seen in the distribution of the gas and the rotation curves at the center of the Milky Way, and in nearby barred galaxies (Blitz, these proceedings, and del Burgo et al., these proceedings).

We propose here a new dynamical mechanism to account for the bar offcentering. It is based on the presence of an $m=1$ density wave, whose first manifestation will be the off-centering of the central region of the galaxy (see also Miller and Smith, 1992, Combes, these proceedings, and Junqueira and Combes, 1996). The mechanism we propose is a non-linear excitation of the $m=1$ perturbation by the strong $m=2$ due to the linearly unstable bar and $m=3$ mode.

\section{Non-linear coupling}

If two spiral waves coexist in a disk, we expect them to excite beat waves at the sum and difference frequencies and wavenumbers: thus in particular an $m=2$ and an $m=3$ spirals, with frequencies $\omega_{2}$ and $\omega_{3}$ create a beat wave with $m=1$ and $\omega_{1}=\omega_{3}-\omega_{2}$. If at this frequency and wavenumber the beat wave obeys the dispersion relation, i.e. can propagate in the disk, it can very efficiently exchange energy and angular momentum with the parent waves. We have already found (see Masset and Tagger, 1997a, and references therein), from analytical and numerical work, a number of examples where this non-linear coupling can play an important role in the 
evolution of spirals and warps. Thus we expect that, in disks where the $m=2$ and $m=3$ spirals are linearly unstable by the classical Swing mechanism, they can excite an $m=1$ wave which could explain the off-centering of the central region of the disk. In order to check this, we have performed numerical simulations with a polar Particle-Mesh code, whose small grid size in the central region allows precise physics and diagnostics.

\section{Results}
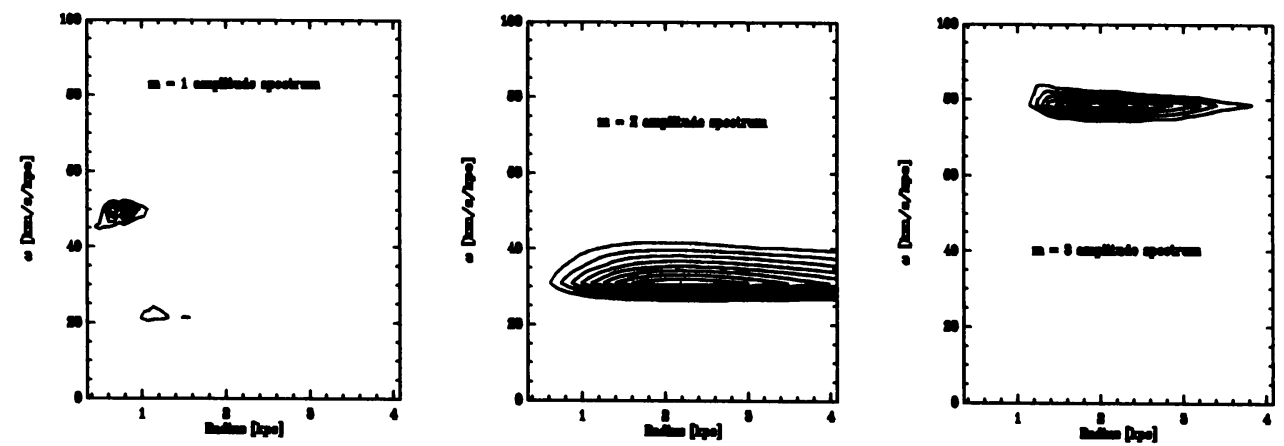

We show above the amplitude spectra (Masset and Tagger, 1997b) of the $m=1, m=2$ and $m=3$ relative perturbed densities for a typical simulation. We see the signature of the bar on the $m=2$ plot, at the frequency $\omega_{2}=30 \mathrm{~km} / \mathrm{s} / \mathrm{kpc}$; on the $m=3$ we see a well defined mode at $\omega_{3}=80 \mathrm{~km} / \mathrm{s} / \mathrm{kpc}$, and on the $m=1$ plot the expected beat wave at $\omega_{1}=80-30=50 \mathrm{~km} / \mathrm{s} / \mathrm{kpc}$. This wave corresponds to a $60 \mathrm{pc}$ deviation of the center of gravity of the inner regions. In order to specify the energy transfer between waves we have redone the simulation by setting to zero at each timestep the $m=1$ potential component, and hence inhibiting possible $m=1$ linear modes. We still see the $m=3$ mode, which proves that it is not excited by the non-linear coupling. When we redo the simulation by setting to zero the $m=3$ potential component, we do not see the $m=1$ : this shows that it needs the other modes to be excited, and hence that it is non-linearly excited by the linearly unstable bar and $m=3$ modes.

As a conclusion, this mechanism naturally leads to an $m=1$ perturbation (i.e. an off-centering) whose amplitude is sufficient to explain the asymmetries typically observed. Work is in progress to include self-gravitating gas and a live bulge in the simulations, for a more detailed description.

\section{References}

Junqueira, S. and Combes, F., $1996:$ A\&A, 312, 703

Masset, F. and Tagger, M., 1997a : A\&A, 318, 747.

Masset, F. and Tagger, M., 1997b : A\&A, 322, 442.

Miller, R.H. and Smith, B.F., 1992 : ApJ, 393,508 